\title{
Design of a phosphor-converted LED based lighting system considering both the lighting and color uniformity
}

\author{
Jinren Yan(10)
}

\begin{abstract}
In this work, a scheme of combining an aspheric reflector and a primary packaging freeform lens in phosphor-converted lighting-emitting diode (pc-LED) based lighting system design was developed. In contrast to most of previous works in which the color and illumination uniformity of the pc-LED lighting systems were improved one by one, the developed scheme can improve both indicators simultaneously. To demonstrate the feasibility of the developed design scheme, an initially designed pc-LED optical model, with an average correlated color temperature (CCT) of about $5000 \mathrm{~K}$, was constructed as the light source of the illumination system. The key ingredient to the color uniformity of the illumination system was investigated at first, and then several instances with the cut-off angle ranging from 40 to 60 degrees were further discussed. Detailed analysis shows: for each case, the CCT variation decreases from over $4000 \mathrm{~K}$ to smaller than $300 \mathrm{~K}$ and the illumination uniformity increases from a very low level to better than $80 \%$ on the target region.
\end{abstract}

Keywords: Light-emitting diode, Non-imaging optics, Illumination, Freeform/aspheric surface

\section{Introduction}

Nowadays, due to excellent characteristics, such as long lifetime, energy saving, environment friendly, and high color rendering, light-emitting diodes (LEDs) have been successfully used in the area of indoor/outdoor lighting, vehicle headlights, backlight of liquid crystal display, landscape lighting, search light, and so forth [1-4]. After years of development, a blue LED chip coated with YAG:Ce phosphor material has became one of the most popular commercial white LED generation approaches [5-12]. The basic principle of this type of white LED is that a portion of blue light emitted from LED chip is absorbed by YAG:Ce phosphor material, which generates yellow light, then the generated yellow light mixes with the remaining blue light, forming the white light. Generally, without exerting special treatment, the angular color uniformity (ACU) of the initially designed phosphor-converted LED (pc-LED) is poor. This is due to

Correspondence: renyj0720@163.com

Department of Optoelectronics and Communication Engineering, Yunnan

Open University (Yunnan Vocational \& Technical College of National Defense Industry), Xuefu Road 113, Kunming 650223, China the fact that blue light rays with different emitting angles undergo different optical paths when passing through the YAG:Ce coating, the longer the optical path is, the more the blue light is depleted, finally, the ratios of blue to yellow light are different when viewing the LED light source from different viewing angles [13-19]. A number of methods to enhance the ACU of the pc-LED, such as using freeform optics or elaborately devising the structure of phosphor coating, had been intensively studied [13-24]. However, these works did not discuss in more details about the illumination uniformity, which is another important issue in general illumination applications. In other words, additional secondary optical elements are needed to redistribute the light energy emitted from LED to further improve the illumination performance of the lighting system.

Compared with the methods of devising the phosphor coating structure, freeform optics approaches seem to be more favorable to improve the optical performance of pc-LED based lighting system [20-24], since it can be used not only to improve the ACU of the system, but also to achieve a uniform illumination on the target

\section{Springer Open}

(c) The Author(s). 2021 Open Access This article is licensed under a Creative Commons Attribution 4.0 International License, which permits use, sharing, adaptation, distribution and reproduction in any medium or format, as long as you give appropriate credit to the original author(s) and the source, provide a link to the Creative Commons licence, and indicate if changes were made. The images or other third party material in this article are included in the article's Creative Commons licence, unless indicated otherwise in a credit line to the material. If material is not included in the article's Creative Commons licence and your intended use is not permitted by statutory regulation or exceeds the permitted use, you will need to obtain permission directly from the copyright holder. To view a copy of this licence, visit http://creativecommons.org/licenses/by/4.0/. 
plane [25-29]. Li et al. [26] developed a modified total internal reflection (TIR) lens to address the ACU issue of pc-LED and commendable color and illumination uniformity was obtained. Lai et al. [27] designed a freeform lens for small-angle lighting and uniform distribution of correlated color temperature (CCT) and illumination was achieved. Prins et al. [28, 29] proposed an inverse method for TIR collimators to achieve a uniform color spot light with prescribed intensity distribution in the far field. However, these works were mainly devised for lighting units with small cut-off angles. Different from above-mentioned studies, a novel design scheme aims to simultaneously address both the illumination and color uniformity of pc-LED based illumination system is developed. The key point of this work is combining a primary packaging freeform lens and an aspheric reflector in illumination system design. These two components can not only modulate the light energy emitted from LED to generate a light spot with uniform illuminance distribution, but also mix the blue and yellow light adequately to make the light spot has a uniform color distribution. In addition, this design scheme can achieve luminaire with a specified cut-off angle within a wide cut-off angle range.

\section{Optical model of pc-LED}

As shown in Fig. 1a, a typical pc-LED consists of a substrate, a packaging lens, and an LED chip coated with phosphor material. The LED light source is immersed in the packaging lens [13-15, 22-24]. A portion of blue light emitted from the LED chip is absorbed by the phosphor material and yellow light is yielded by photoluminescence effect, then the yellow light mixes with the remaining blue light to produce white light. Fig. 1b shows the excitation and emission spectra of the phosphor material, and spectrum of the blue LED that used to construct the optical model of the pc-LED in this work [30]. It is assumed that the LED chip has a dimension of $0.7 \mathrm{~mm} \times 0.7 \mathrm{~mm}$ and its average refractive index is 2.5 , the light rays were emitted from the top surface of the LED chip; the phosphor layer was coated on the LED chip and its dimension is $1.0 \mathrm{~mm} \times 1.0 \mathrm{~mm}$, the phosphor particles were approximated as spheres with diameters vary in the range of 1 to $15 \mathrm{um}$; the phosphor powder was mixed with a transparent material with a refractive index of 1.5 , the material of the packaging lens is polymethyl methacrylate (PMMA) with a refractive index of 1.49. More details about the construction of pcLED can be found in [22, 31-33].

Figure 2a shows the simulated real color light pattern of the pc-LED with an average CCT of about $5000 \mathrm{~K}$ and Fig. $2 \mathrm{~b}$ shows the corresponding illuminance and CCT distributions across centre section $(u=0)$ of the light pattern. One can find out easily that the CCT in the centre region is larger than that in the edge and more than $4000 \mathrm{~K}$ CCT difference can be noticed from centre to edge. Furthermore, a yellow light ring can be seen around the pattern, the simulation result is similar to the results shown in the previous works $[14,15,21-$ 24, 31, 32].

Figure 3 shows the light intensity distribution curve of the modeled pc-LED and it illustrates that the light source is close to an ideal Lambertian emitter. For the purpose of obtaining precise simulation results in the following, the light intensity distribution of the pc-LED was represented by a polynomial function $[34,35]$, as expressed in eq. (1):

$$
I_{s}(\phi)=I_{0}\left(a_{0}+a_{1} \phi^{2}+a_{2} \phi^{4}+a_{3} \phi^{6}+a_{4} \phi^{8}\right)
$$

where $\phi$ represents the angle that the emitted light ray makes with the optical axis (the line vertical to the top

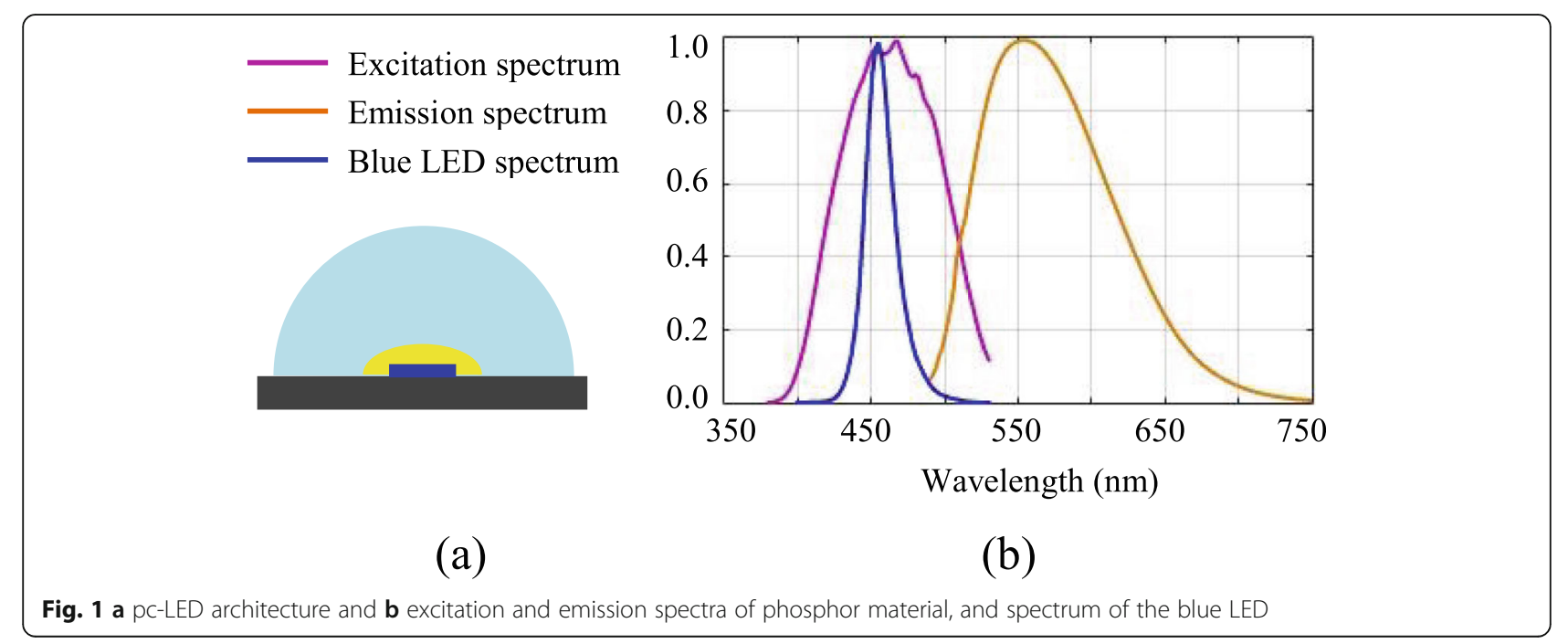




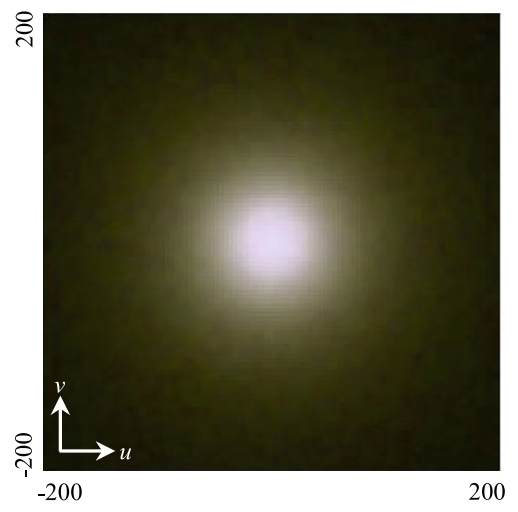

(a)

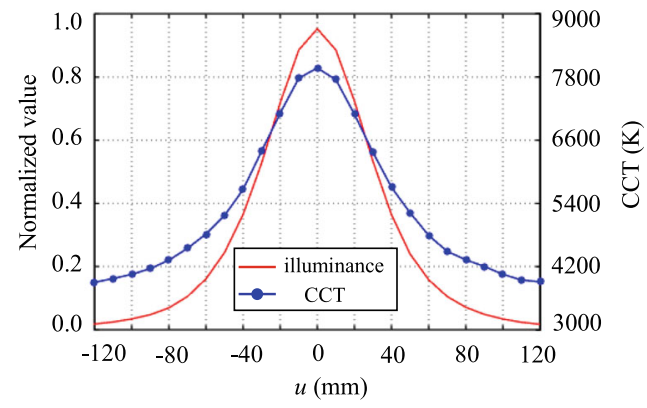

(b)

Fig. 2 a Real color light pattern of the modeled LED light source with an average CCT of about $5000 \mathrm{~K}$, and $\mathbf{b}$ shows the corresponding illuminance and CCT distributions across centre section $(u=0)$

surface of LED chip). $I_{0}$ is the luminous intensity at $\phi=$ $0^{\circ} . a_{i}(i=0,1,2,3,4)$ represent the coefficients of the polynomial function and the numeric values are $\mathrm{a}_{0}=$ $0.9707, \mathrm{a}_{1}=-0.6318, \mathrm{a}_{2}=0.0536, \mathrm{a}_{3}=0.05509, \mathrm{a}_{4}=-$ 0.01422 , respectively.

\section{Design method}

Figure 4 shows the geometrical structure of the developed LED luminaire. The primary packaging lens, into which a pc-LED light source is immersed, is a freeform lens that made by PMMA optical material [22, 23]. It is assumed that the LED source is placed at the origin of the coordinates (represent by $(u, v, z)$ and $v$ is in the outward direction perpendicular to the plane). The aspheric reflector is made by metallic material and the inside surface of it is coated with reflective material with a reflectivity of $95 \%$. $a$ and $b$ represent the semi-aperture size and the depth of the reflector, respectively. $\phi_{c u t}$ denotes the cut-off angle of the luminaire, it is generally given by designers according to illumination requirements. $\left(u_{l}\right.$, $\left.z_{l 0}\right)$ and $\left(u_{r 0}, z_{r 0}\right)$ represent the initial points of the freeform lens and the aspheric reflector, respectively. $\left(u_{l 0}\right.$,

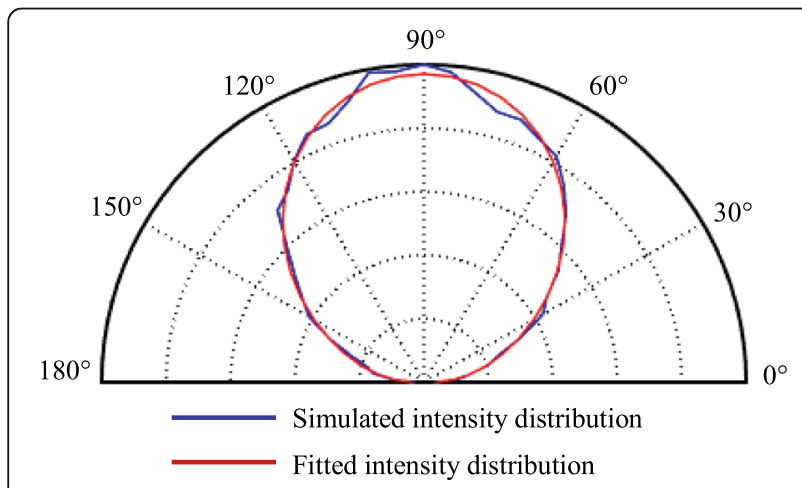

Fig. 3 Simulated and fitted light intensity distribution curves of the modeled LED source $z_{l 0}$ ) divides the surface of the lens into two parts: surface 1 (S1) and surface 2 (S2). It is worth noting that S1 and $\mathrm{S} 2$ are connected smoothly at the connecting point $\left(u_{l 0}\right.$, $\left.z_{l o}\right)$. The angle $\phi_{c}$ divides the light energy emitted from the LED into two parts: light energy in the angle range of $0^{\circ}$ to $\phi_{c}$ and in the range of $\phi_{c}$ to $\pi / 2$. The S1 of the freeform lens was designed to deflect the light rays in the range of $0^{\circ}$ to $\phi_{c}$ toward the range of $0^{\circ}$ to $\phi_{c u t}$ to generate a light pattern with uniform light distribution on the target surface. In order to generate a light pattern

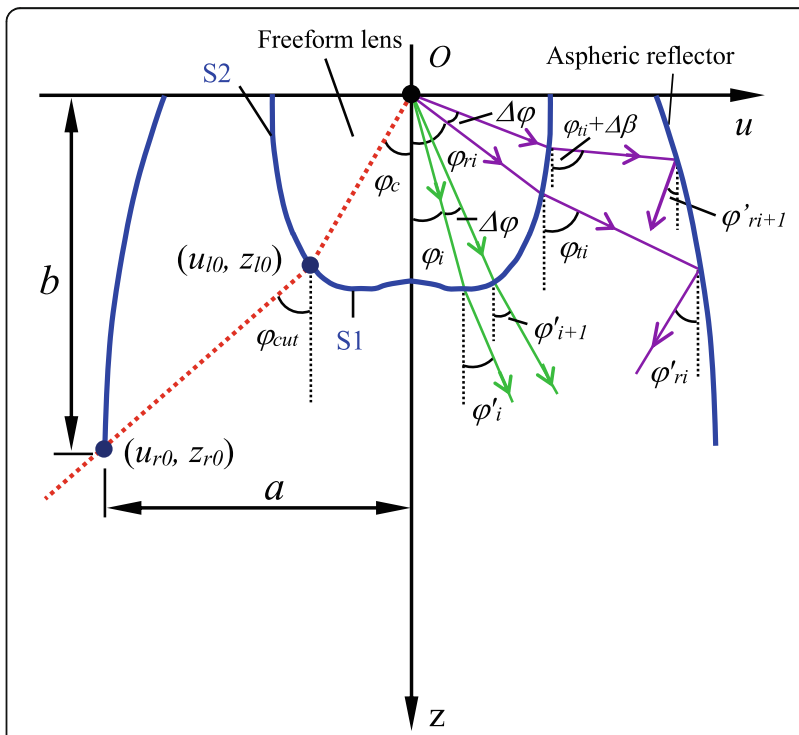

Fig. 4 The structure of the developed LED luminaire. $a$ and $b$ represent the semi-aperture size and the depth of the reflector, respectively; $\varphi_{\text {cut }}$ represents the cut-off angle of the luminaire, $\left(u_{10}, z_{10}\right)$ and $\left(u_{r_{0}}, z_{00}\right)$ represent the initial points of the freeform lens and the aspheric reflector, respectively; $\left(u_{10}, Z_{10}\right)$ divides the surface of the lens into two parts: surface 1 (S1) and surface 2 (\$2), the two surfaces are connected smoothly at the connecting point; $\varphi$, with subscript, or both superscript, denotes the angle that light ray makes with optical axis, $\Delta \varphi$ and $\Delta \beta$ denote the angle increment of emitting and refractive light ray, respectively 


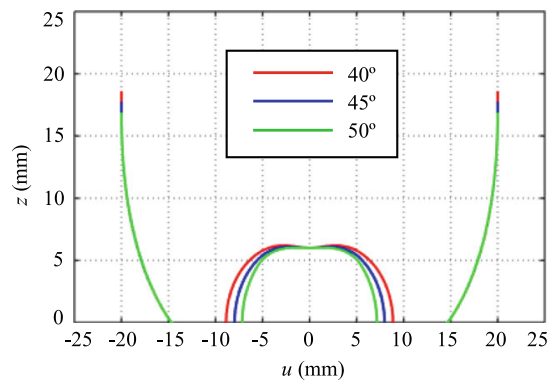

(a)

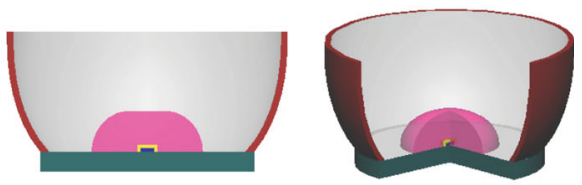

(b)

Fig. 5 a Profiles of the designed luminaires with different $\varphi_{c}$ and $\mathbf{b}$ the three dimension model of the luminaire with $\varphi_{c}=45^{\circ}$

with uniform color distribution on the target surface, it is important to mix the yellow and blue light adequately. Thus, the $\mathrm{S} 2$ of the lens was designed to deflect the light rays in the angle range of $\phi_{c}$ to $\pi / 2$ toward the aspheric reflector, and then the reflector reflected these rays to the range of $\phi_{c u t}$ to $0^{\circ}$ to generate a light pattern as the previous one. The aforementioned contents imply that the light distribution on the target region was formed by two uniformly distributed light patterns, which can be mathematically expressed as:

$$
\begin{aligned}
& 2 \pi \int_{0}^{\phi_{c}} I_{s}(\phi) \sin \phi d \phi=2 \pi \int_{0}^{\phi_{\text {cut }}} I_{l s}(\phi) \sin \phi d \phi \\
& 2 \pi \rho \int_{\phi_{c}}^{\pi / 2} I_{s}(\phi) \sin \phi d \phi=2 \pi \int_{0}^{\phi_{c u t}} I_{l s}(\phi) \sin \phi d \phi
\end{aligned}
$$

where $I_{s}(\phi)$ and $I_{l s}(\phi)$ denote the light intensity distributions of the LED light source and the designed luminaire, respectively. $\rho$ denotes the reflectivity of the reflective material. For a uniform far-field illumination, the light intensity distribution of the lighting unit can be expressed as $[35,36]$ :

$$
I_{l s}(\phi)=\frac{I_{l 0}}{\cos ^{3}(\phi)}
$$

where $I_{l o}$ denotes the luminous intensity of the lighting unit at $\phi=0^{\circ}$.

In order to construct the freeform lens and the aspheric reflector, the relations between the incident rays and the outgoing rays at the two surfaces should be obtained. For the light ray with the emitting angle $\phi_{i}$ within the angle range of $0^{\circ}$ to $\phi_{c}$, since it only passes through the freeform lens, the relation between the emitting ray angle $\phi_{i}$ and the corresponding outgoing ray angle $\phi_{i}^{\prime}$ at the lens surface can be obtained by eq. (5). For the light ray with emitting angle $\phi_{r i}$ within the angle range of $\phi_{c}$ to $\pi / 2$, since it is first refracted by the freeform lens and subsequently reflected by the aspheric reflector, the relation between the emitting ray angle $\phi_{r i}$ and the corresponding reflected outgoing ray angle $\phi_{r i}^{\prime}$ can be obtained by eq. (6).

$$
\frac{2 \pi \int_{0}^{\phi_{c u t}} I_{l s}(\phi) \sin \phi d \phi}{2 \pi \int_{0}^{\phi_{c}} I_{s}(\phi) \sin \phi d \phi}=\frac{2 \pi \int_{0}^{\phi^{\prime} i} I_{l s}(\phi) \sin \phi d \phi}{2 \pi \int_{0}^{\phi_{i}} I_{s}(\phi) \sin \phi d \phi}
$$

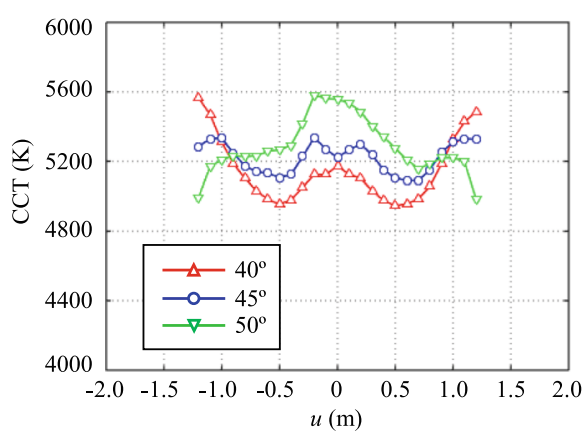

(a)

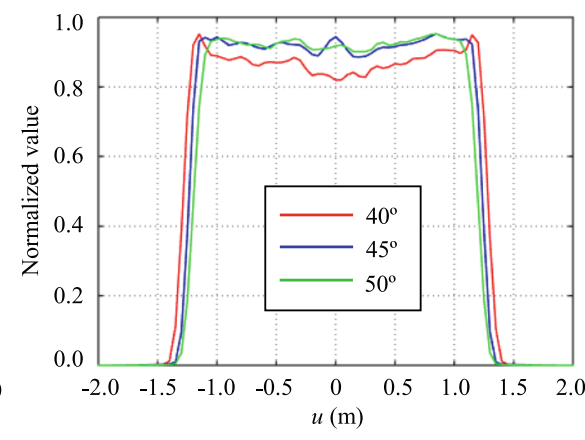

(b)

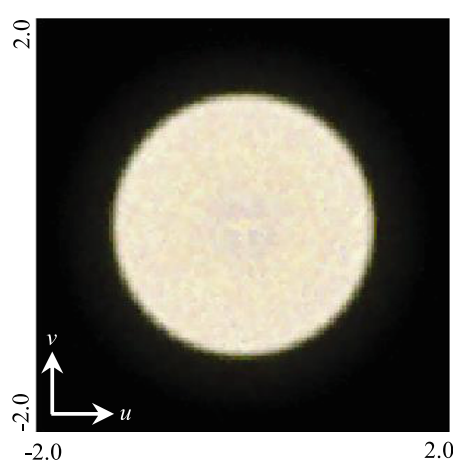

(c)

Fig. 6 Simulation results of the luminaires with different energy partition angle $\varphi_{c}$ (a) CCT distribution, (b) illuminance distributions, and (c) simulated real color pattern of the luminaires with $\varphi_{c}=45^{\circ}$ 
Table 1 Simulation results of the luminaires with different energy partition angle $\varphi_{c}$ and single lens

\begin{tabular}{|c|c|c|c|c|c|}
\hline Structure & $\varphi_{\text {cut }}$ & $\varphi_{c}$ & $\triangle C C T(K)$ & $2 a(\mathrm{~mm}) \times b(\mathrm{~mm})$ & illumination uniformity \\
\hline \multirow[t]{2}{*}{ Developed } & & $40^{\circ}$ & 620 & $40.00 \times 18.51$ & $90 \%$ \\
\hline & $50^{\circ}$ & $45^{\circ}$ & 246 & $40.00 \times 17.63$ & $88 \%$ \\
\hline \multirow[t]{2}{*}{ Single Lens } & & $50^{\circ}$ & 598 & $40.00 \times 16.78$ & $85 \%$ \\
\hline & $50^{\circ}$ & - & 5628 & - & $84 \%$ \\
\hline
\end{tabular}

$$
\frac{2 \pi \int_{0}^{\phi_{c u t}} I_{l s}(\phi) \sin \phi d \phi}{2 \pi \rho \int_{\phi_{c}}^{\pi / 2} I_{s}(\phi) \sin \phi d \phi}=\frac{2 \pi \int_{0}^{\phi^{\prime} r i} I_{l s}(\phi) \sin \phi d \phi}{2 \pi \rho \int_{\phi_{r i}}^{\pi / 2} I_{s}(\phi) \sin \phi d \phi}
$$

Up to present, the only unknown quantity in construction of the two surfaces is $\phi_{t i}$, as shown in Fig. 4, which represents the refraction angle of outgoing light ray corresponding to the incident light ray with emitting angle larger than $\phi_{c}$. Keeping in mind that the emitting angle $\phi_{r i}$ of the light ray is increased step by step by a small angle increment $\Delta \phi$ in constructing the freeform lens, thus, it is instinctively assumed that the corresponding outgoing ray angle $\phi_{t i}$ is also increased step by step by a small increment angle $\Delta \beta=\left(\pi / 2-\phi_{c}\right) / M$, where $M$ is a positive integer which denotes the number of partitions. As $\phi_{t i}=\phi_{c}+i \Delta \beta$ is known, together with the emitting light ray angle $\phi_{i}$ and $\phi_{r i}$, refracted outgoing ray angle $\phi_{i}^{\prime}$, reflected outgoing ray angle $\phi_{r i}^{\prime}$, and the given initial points of the freeform lens and aspheric reflector, the lens and reflector can be constructed easily [34, 37].

\section{Simulation and discussion}

\section{Influence of $\varphi_{c}$ on color uniformity}

Figure 2 shows that both the illuminance and the CCT distributions decrease nonlinearly from centre to edge on the receiver as a bare LED light source is used in the illumination system. To design a system with acceptable optical performance using this white LED source, both the illumination and color uniformity should be considered in the design process. The uniformity of lighting on the target surface is ensured by eqs. (5) and (6), the dependence of color uniformity on $\phi_{c}$ will be discussed in the following. In this section, the cut-off angle $\phi_{c u t}$ of the luminaire is set to $50^{\circ}$, and $\phi_{c}$ is changed from $40^{\circ}$ to $50^{\circ}$. As the initial points $u_{l 0}$ of the lens and $u_{r 0}$ of the reflector are given, the illumination system can be constructed. In order to facilitate comparison, $u_{l o}$ was selected to make the centre height of the lens to be $6 \mathrm{~mm}$ for each $\phi_{c}$, and $u_{r 0}$ was set to $20 \mathrm{~mm}$. Figure $5 \mathrm{a}$ and $\mathrm{b}$ show the profiles of the designed luminaires with different energy partition angle $\phi_{c}$ and the three dimension model of the luminaire with $\phi_{c}=45^{\circ}$, respectively. One can note from the figure that the shapes of the aspheric reflectors corresponding to different energy partition angle $\phi_{c}$ are almost identical and the small difference in the geometrical sizes of the reflectors is caused by the difference in selection of $u_{l 0}$ of the lens. One can also notice from the figure that as the centre height of the lens remains constant, the total geometrical size of the freeform lens decreases with the increase of $\phi_{c}$.

Figure 6 shows the simulation results of the designed luminaires with different energy partition angle $\phi_{c}$. It is noted that the lines shown in Fig. $6 \mathrm{a}$ and $\mathrm{b}$ are sampled values across the centre $(u=0)$ of the target surface, and

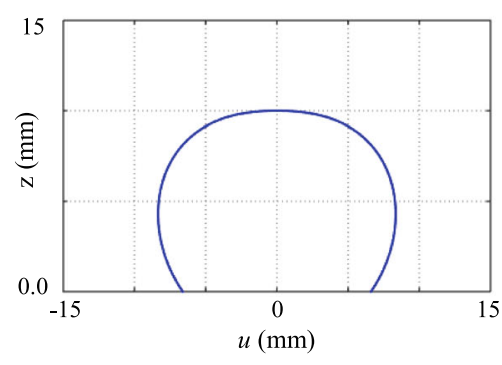

(a)

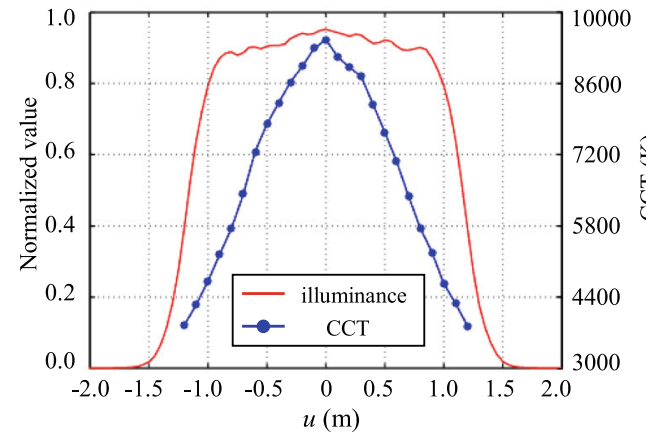

(b)

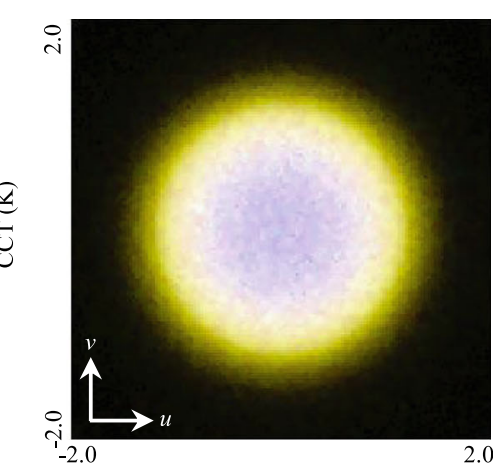

(c)

Fig. 7 Simulation results of the luminaire with single freeform lens (a) profile of the lens, (b) illuminance and CCT distributions, (c) real color light pattern 


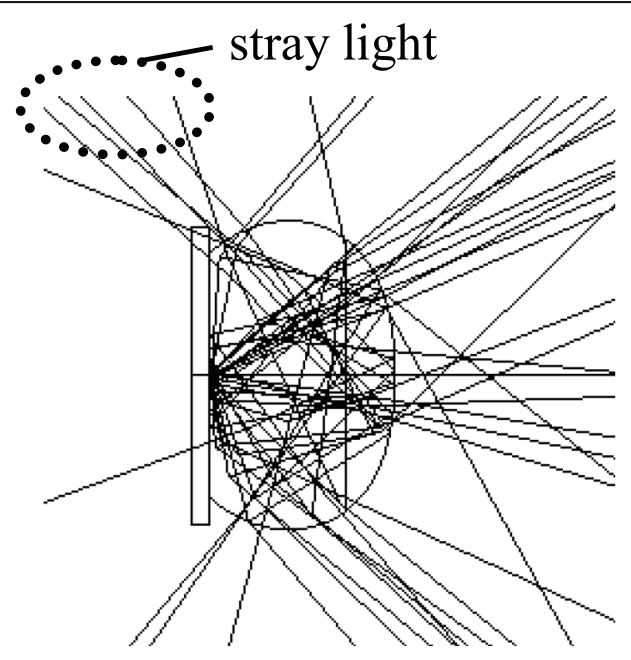

(a) without stray light

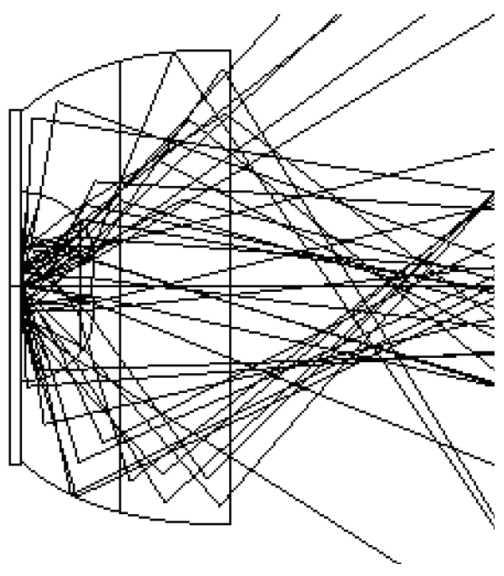

(b)

Fig. 8 Ray tracing chart of (a) single freeform lens and (b) developed design scheme. Undesired stray light is unavoidable by only a single freeform lens, but it can be eliminated by the aspheric reflector of the novel system

more detailed simulation results are shown in Table 1. It can be found from Fig. 6a that for a large energy partition angle $\phi_{c}$, the CCT in the centre region of the target surface is larger than that in the edge; on the contrary, the CCT in the centre is smaller than that in the edge for a small $\phi_{c}$. This result can be interpreted with the help of Fig. 2, one can note from the figure that the ratio of blue to yellow light in the centre is larger than that in the edge, since the light energy in the range of $0^{\circ}$ to $\phi_{c}$ and $\phi_{c}$ to $\pi / 2$ separately generate a uniform light pattern with same geometric size on the target surface, as $\phi_{c}$ increases, the quantity of yellow light reflected to the centre decreases, the CCT in the centre increases; conversely, the CCT in the centre decreases. As shown in Table 1 , when $\phi_{c}=45^{\circ}$, the variation of CCT across the centre of the target surface is smaller than $300 \mathrm{~K}[8,38]$.
Figure $6 \mathrm{~b}$ shows illuminance distribution corresponding to different energy partition angle $\phi_{c}$, one can note that uniform illumination was obtained for each case; however, a very small difference exists in the extension of the illuminance distribution among these three cases, the reason is that the geometrical size ratios of the lens to the reflector are different for the three energy partition angle $\phi_{c}$, as shown in Fig. 5a. Figure 6c shows the simulated real color light pattern of the luminaire with $\phi_{c}=45^{\circ}$, one can note that a uniform illumination, along with a uniform color distribution, was obtained.

Using the pc-LED source, an illumination system with a single freeform lens was also designed for comparison, as shown in Fig. 7. Figure 7a shows the profile of the single freeform lens, and the corresponding simulation results are shown in Fig. $7 \mathrm{~b}$ and c. It can be found from

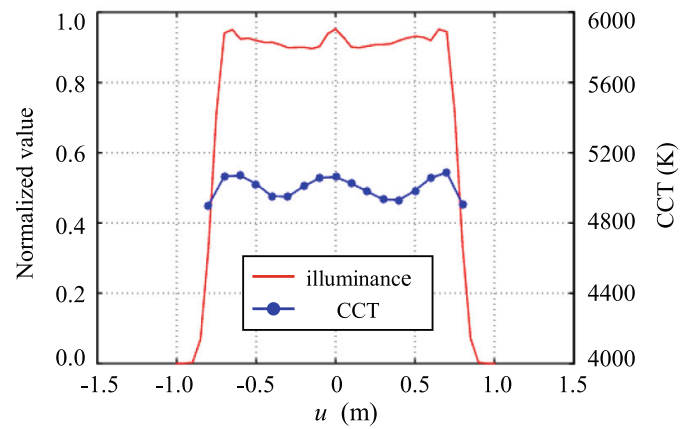

(a)

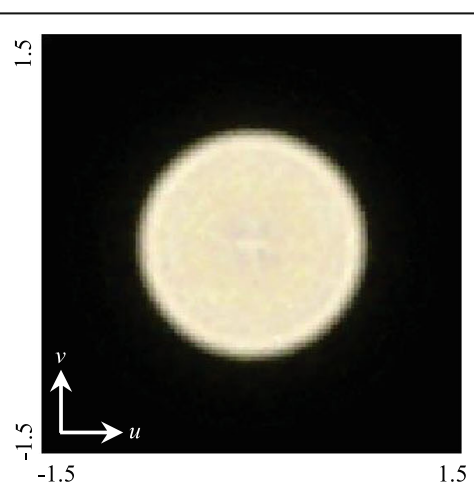

(b)

Fig. 9 Simulation results of the luminaire with the cut-off angle of $40^{\circ}$ (a) illuminance and CCT distributions, (b) real color light pattern 


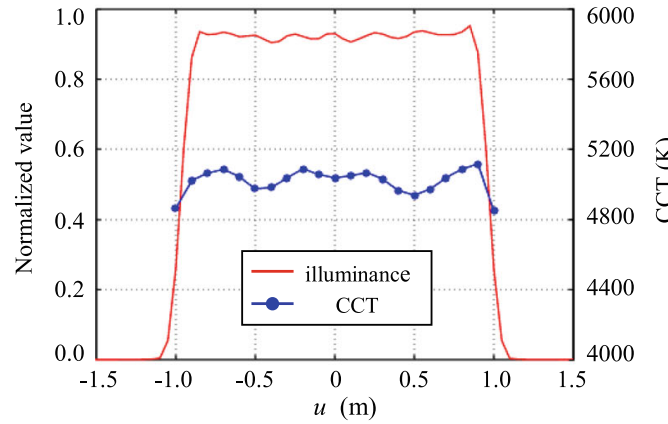

(a)

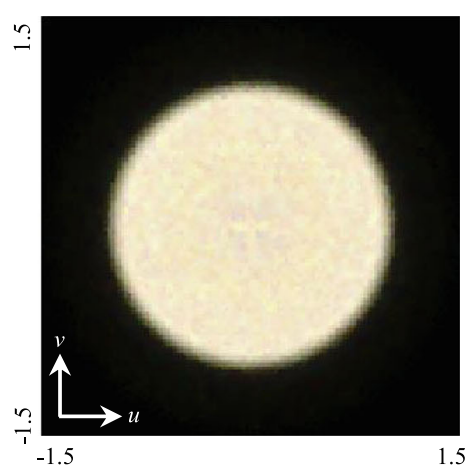

(b)

Fig. 10 Simulation results of the luminaire with the cut-off angle of $45^{\circ}$ (a) illuminance and CCT distributions, (b) real color light pattern

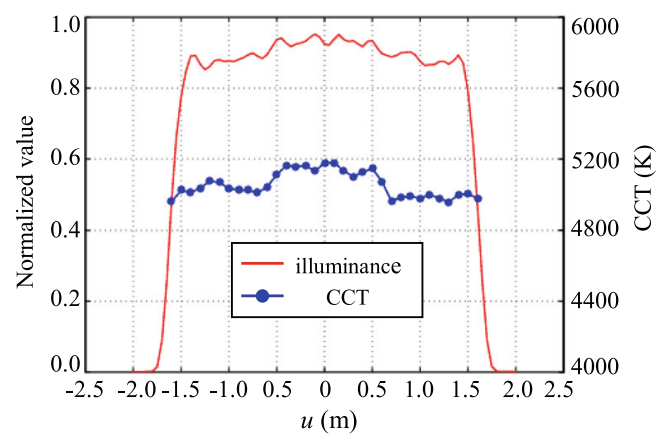

(a)

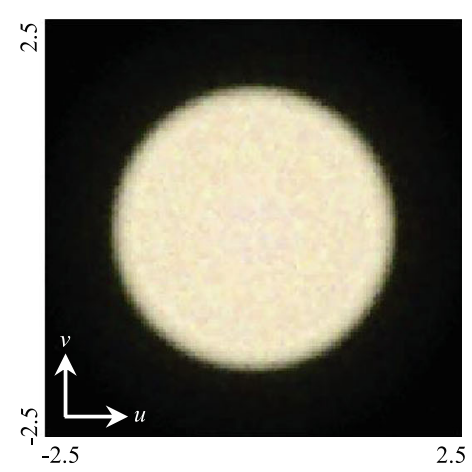

(b)

Fig. 11 Simulation results of the luminaire with the cut-off angle of $55^{\circ}$ (a) illuminance and CCT distributions, (b) real color light pattern

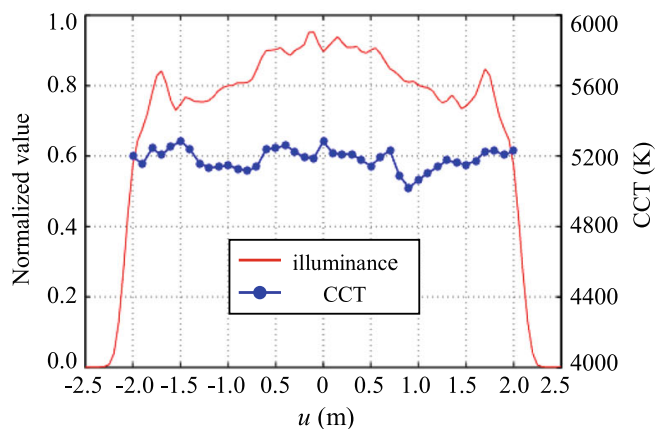

(a)

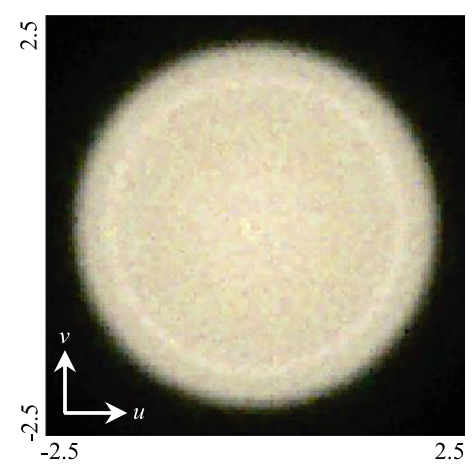

(b)

Fig. 12 Simulation results of the luminaire with the cut-off angle of $60^{\circ}$ (a) illuminance and CCT distributions, (c) real color light pattern 
Table 2 Simulation results of the designed luminaires with different cut-off angle $\varphi_{\text {cut }}$

\begin{tabular}{lllll}
\hline $\boldsymbol{\varphi}_{\text {cut }}$ & $\boldsymbol{\varphi}_{\boldsymbol{c}}$ & $\boldsymbol{\Delta C C T}(\boldsymbol{K})$ & $\boldsymbol{2} \boldsymbol{a}(\mathbf{m m}) \times \boldsymbol{b}(\mathbf{m m})$ & illumination uniformity \\
\hline $40^{\circ}$ & $52^{\circ}$ & 186 & $40.00 \times 23.84$ & $89 \%$ \\
$45^{\circ}$ & $50^{\circ}$ & 264 & $40.00 \times 20.00$ & $90 \%$ \\
$55^{\circ}$ & $43^{\circ}$ & 219 & $40.00 \times 16.09$ & $87 \%$ \\
$60^{\circ}$ & $40^{\circ}$ & 265 & $60.00 \times 20.84$ & $82 \%$ \\
\hline
\end{tabular}

Fig. 7b that although an uniform illumination is obtained on the target surface, the color distribution is very terrible, the CCT in the centre is higher than that in the edge and about $5600 \mathrm{~K} \mathrm{CCT}$ variation can be noted from the centre to edge. Such big color variation is unacceptable in most of applications since uncomfortable visual effect will disturb human beings, as shown in Fig. 7c. It seems that the novel developed system has an additional reflector compared with single freeform lens based system, in fact, in most of applications, a reflector is an indispensable component of the illumination system, since it can be used not only to adjust light beams, but also to eliminate undesired stray light and/or to protect the light source. As shown in Fig. 8, without reflector, undesired stray light was generated by the single freeform lens, in contrast, the stray light can be blocked by the aspheric reflector of the novel system.

\section{Design with different cut-off angle $\varphi_{\text {cut }}$}

In different illumination applications, the luminaires with different cut-off angles are likely to be required, several examples within the cut-off angle range of $40^{\circ}$ to $60^{\circ}$ were designed. Figures. 9, 10, 11, and 12 show the simulation results of the luminaires with the cut-off

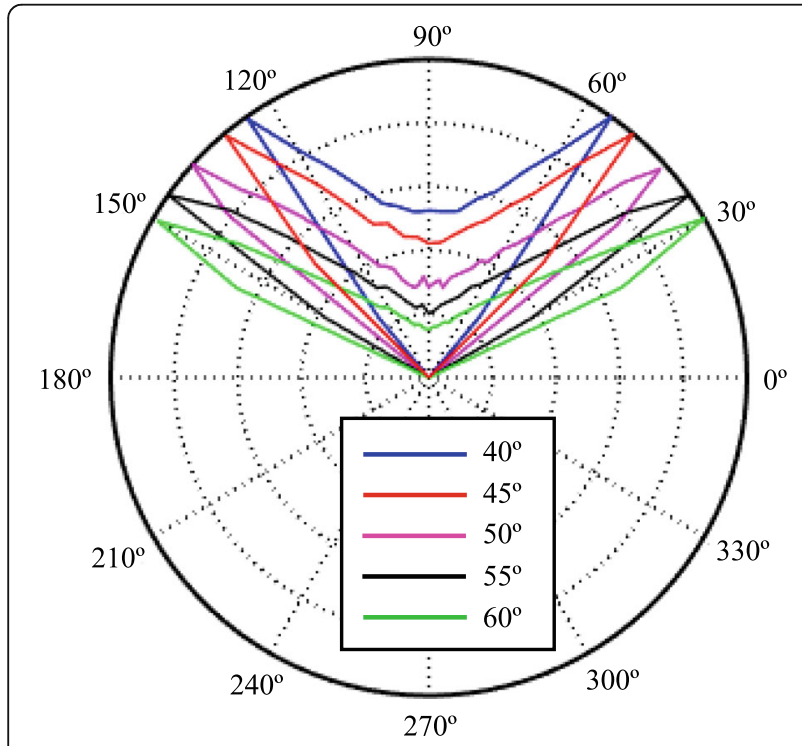

Fig. 13 Light intensity distribution of the designed luminaires angle of $40^{\circ}, 45^{\circ}, 55^{\circ}$, and $60^{\circ}$, respectively. Detailed simulation results were concluded in Table 2. After checking the simulation results, one can note that the energy partition angle $\phi_{c}$ decreases with the increase of $\phi_{\text {cut }}$, one can also found that for each case, the illumination uniformity is better than $80 \%$, the CCT variation is less than $300 \mathrm{~K}$. These indices are within acceptable levels $[8,34,38]$. It is noted that the uniformity of illumination in this work is defined as the ratio of minimum illuminance to the average illuminance on the target surface. Figure 13 shows the light distribution curves of the designed luminaires with different cut-off angles, it can be seen that batwing-like distribution curves were obtained for all cases.

\section{Conclusion}

In this study, a simple design scheme which can address both the problem of illumination and color uniformity of pc-LED based illumination system has been discussed. By employing only two ordinary optical components, i.e., a primary packaging lens and a reflector, the scheme can improve both the illumination and color performance of a pc-LED based lighting unit. Simulation results show that as proper energy partition angle $\phi_{c}$ was selected for a pre-specified $\phi_{\text {cut }}$, better than $80 \%$ illumination uniformity, smaller than $300 \mathrm{~K} \mathrm{CCT}$ variation can be obtained. Since the ACU of a pc-LED source mainly depends on the thickness variation of the phosphor layer, the design scheme showed in this study can increase the tolerances in coating the phosphor layer and reduce the cost of the illumination system to some extent. It is a useful alterative design method to improve the optical performance of pc-LED based lighting system in lighting industry. However, this study only focuses on the issue of illumination and color uniformity for a point-like pc-LED based illumination system, further extension to extended pc-LED based illumination system is required in the future work and this study is an important building block for more in-depth research.

\section{Abbreviations}

PC: Phosphor-converted; LED: Light-emitting diode; CCT: Correlated color temperature; ACU: Angular color uniformity; TIR: Total internal reflection; PMMA: Polymethyl methacrylate

\section{Acknowledgements \\ The author thanks Dr. Ting Wang from Yunnan University, for he valuable suggestions and selfless help that have helped improve this manuscript substantially.}

\section{Author's contributions}

This work was done by the corresponding author. The author(s) read and approved the final manuscript.

\section{Funding}

This work was sponsored by the Scientific Research Fund of Yunnan Provincial Education Department under grant No.2019 J1151. 


\section{Availability of data and materials}

All data generated during this work are included in the article.

\section{Declarations}

\section{Competing interests}

The author declares that there is no conflict of interest regarding this paper.

Received: 1 February 2021 Accepted: 16 June 2021

Published online: 30 June 2021

\section{References}

1. Crawford, M.H.: LEDs for solid-state lighting: performance challenges and recent advances. IEEE J. Quantum Elect. 15(4), 1028-1040 (2009). https://doi. org/10.1109/JSTQE.2009.2013476

2. Kong, H.S., Ibbetson, J., Edmond, J.: Status of GaN/sic-based LEDs and theire application in solid state lighting. Phys. Status Solidi. C. 11(3-4), 621-623 (2014). https://doi.org/10.1002/pssc.201300458

3. Kudsieh, N., Bhutta, M.K., Raja, M.Y.A.: High power LED assemblies for solid state lighting - thermal analysis. Optik. 126(22), 3452-3456 (2015). https:// doi.org/10.1016/j.ijleo.2015.07.054

4. Rubinger, R.M., da Silva, E.R., Pinto, D.Z., Rubinger, C.P.L., Oliveira, A.F., da Bortoni, E.C.: Comparative and quantitative analysis of white light-emitting diodes and other lamps used for home illumination. Opt. Eng. 54(1), 014104 (2015)

5. Luo, H., Kim, J.K., Schubert, E.F., Cho, J., Sone, C., Park, Y.: Analysis of highpower packages for phosphor-based white-light-emitting diodes. Appl. Phys. Lett. 86(24), 243505 (2005). https://doi.org/10.1063/1.1949282

6. Zhu, Y.T., Narendran, N., Gu, Y.M.: Investigation of the Optical Properties of YAG: Ce Phosphor. Proc SPIE. 6337, 63370S (2006)

7. Chen, C.Y., Yang, T.H., Hsu, C.H., Sun, C.C.: High-efficiency white LED packaging with reduced phosphor concentration. IEEE Photonic. Tech. L. 25(7), 694-696 (2013). https://doi.org/10.1109/LPT.2013.2248141

8. Sun, C.C., Chen, C.Y., Chang, J.H., Yang, T.H., Ji, W.S., Wu, H.M.: Linear calculation model for prediction of color rendering index performance associated with correlated color temperature of white light emitting diodes with two phosphors. Opt. Eng. 5(15), 054003 (2012)

9. Ying, S.P., Shen, J.Y.: Concentric ring phosphor geometry on the luminous efficiency of white-light-emitting diodes with excellent color rendering property. Opt. Lett. 41(9), 1989-1992 (2016). https://doi.org/10.1364/OL.41. 001989

10. Ying, S.P., Chien, Y.Y.: Effect of reassembled remote phosphor geometry on the luminous efficiency and spectra of white light emitting diodes with excellent color rendering property. IEEE T. Electron Dev. 63(3), 1117-1121 (2016). https://doi.org/10.1109/TED.2016.2516563

11. Tran, N.T., Shi, F.G.: Studies of phosphor concentration and thickness for phosphor-based white light-emitting diodes. J. Lightwave Technol. 26(21), 3556-3559 (2008). https://doi.org/10.1109/JLT.2008.917087

12. Zhang, X.R., Zhang, S., Wang, G.D., Wang, Y.Y., Liang, M., Zhang, T., Wang, J.X. , Li, J.M., Liu, Z.Q., Yi, X.Y.: Near ultraviolet chip based phosphor-converted soloar -spectrum white light-emitting diode. Opt. Eng. 59(1), 015104 (2020)

13. Liu, Z.Y., Liu, S., Wang, K., Luo, X.B.: Studies on optical consistency of white LEDs affected by phosphor thickness and concentration using optical simulation. IEEE T. Compon. Pack. T. 33(4), 680-687 (2010). https://doi.org/1 $0.1109 /$ TCAPT.2010.2044576

14. Liu, Z.Y., Liu, S., Wang, K., Luo, X.B.: Optical analysis of color distribution in white LEDs with various packaging methods. IEEE Photonic. Tech. L. 20(24), 2027-2029 (2008)

15. Sun, C.C., Chen, C.Y., Chen, C.C., Chiu, C.Y., Peng, Y.N., Wang, Y.H., Yang, T.H., Chung, T.Y., Chung, C.Y.: High uniformity in angular correlated-colortemperature distribution of white LEDs from $2800 \mathrm{~K}$ to $6500 \mathrm{~K}$. Opt. Express. 20(6), 6622-6630 (2012). https://doi.org/10.1364/OE.20.006622

16. Cheng, T., Yu, X.J., Ma, Y.P., Xie, B., Chen, Q., Hu, R., Luo, X.B.: Angular color uniformity enhancement of white LEDs by les wetting phosphor coating. IEEE Photonic. Tech. L. 28(14), 1589-1592 (2016). https://doi.org/10.1109/ LPT.2016.2554631

17. Sommer, C., Hartmann, P., Pachler, P., Schweighart, M., Tasch, S., Leising, G Wenzl, F.: A detailed study on the requirements for angular homogeneity of phosphor converted high power white LED light sources. Opt. Mater. 31(6), 837-848 (2009). https://doi.org/10.1016/j.optmat.2008.09.009
18. Huang, H.T., Tsai, C.C., Huang, Y.P.: Conformal phosphor coating using pulsed spray to reduce color deviation of white LEDs. Opt. Express. 18(52) A201-A206 (2010). https://doi.org/10.1364/OE.18.00A201

19. Zheng, H., Luo, X.B., Hu, R., Cao, B., Fu, X., Wang, Y.M., Liu, S.: Conformal phosphor coating using capillary microchannel for controlling color deviation of phosphor-converted white light-emitting diodes. Opt. Express. 20(5), 5092-5098 (2012). https://doi.org/10.1364/OE.20.005092

20. Chen, J.C., Fritz, B., Liang, G.W., Ding, X.R., Lemmer, U., Gomard, G.: Microlens arrays with adjustable aspect ratio fabricated by electrowetting and their application to correlated color temperature tunable light-emitting diodes. Opt. Express. 27(4), A25-A38 (2019). https://doi.org/10.1364/OE.27.000A25

21. Lee, T.X., Lu, T.L., Chen, B.S.: Combining near-field hyperspectral imaging and far-field spectral-angular distribuion to develop mid-field white LED optical models with spatial color deviation. Opt. Express. 24(14), A1094-A1106 (2016). https://doi.org/10.1364/OE.24.0A1094

22. Wang, K., Wu, D., Chen, F., Liu, Z.Y., Luo, X.B., Liu, S.: Angular color uniformity enhancement of white light emitting diodes integrated with freeform lenses. Opt. Lett. 35(11), 1860-1862 (2010). https://doi.org/10.1364/OL.35.001860

23. Li, S.M., Wang, K., Chen, F., Liu, S.: New freeform lenses for white LEDs with high color spatial uniformity. Opt. Express. 20(22), 24418-24428 (2012). https://doi.org/10.1364/OE.20.024418

24. Zhu, L., Wang, X.H., Lai, P.T., Choi, H.W.: Angularly uniform white lightemitting diodes using an integrated reflector cup. IEEE Photonic. Tech. L. 22(7), 513-515 (2010). https://doi.org/10.1109/LPT.2010.2041651

25. Zhao, Z.L., Zhang, H.H., Liu, S., Wang, X.Z.: Effective freeform TIR lens designed for LEDs with high angular color uniformity. Appl. Opt. 57(15), 4216-4221 (2016)

26. Li, S.M., Chen, F., Wang, K., Zhao, S., Zhao, Z.L., Liu, S.: Design of a compact modified total internal reflection lens for high angular color uniformity. Appl. Opt. 51(36), 8557-8562 (2012). https://doi.org/10.1364/AO.51.008557

27. Lai, J., Li, X.F., Ge, P.: Freeform lens design for small-angle lighting with uniform distribution of correlated color temperature and illumination. Optik. 168, 800-806 (2018). https://doi.org/10.1016/j.ijleo.2018.05.006

28. Prins, C., ten Thije Boonkkamp, J., Tukker, T., IJzerman, W.: An inverse method for color unifomtiy in white LED spotlights. J. Math. Indus. 4(5), 119 (2014)

29. Prins, C.R., ten Thije Boonkkamp, J.H.M., Tukker, T.W., IJzerman, W.L.: An inverse method for the design of TIR collimators to achieve a uniform color light beam. J. Eng. Math. 81(1), 177-199 (2013). https://doi.org/10.1007/s1 0665-012-9584-7

30. Liu, Z.Y., Liu, S., Wang, K., Luo, X.B.: Measurement and numerical studies of optical properties of YAG: Ce phosphor for white light-emitting diode packaging. Appl. Opt. 49(2), 248-257 (2010)

31. Che, Z., Zhang, J., Yu, X.Y., Chen, Z:: Optical design and research on phosphors for white LED. Chinese J. Lumin. 36(10), 1220-1226 (2015) (in Chinese)

32. Qian, K.Y., Ma, J., Fu, W., Luo, Y.: Research on scattering properties of phosphor for high power white light emitting diode based on Mie scattering theory. Acta Phys. Sin-Ch Ed. 61(20), 204201 (2012)

33. Liu, Z.Y., Liu, S., Wang, K., Luo, X.B.: Optical analysis of phosphor's location for high-power light-emitting diodes. IEEE T. D evice Mat. Re. 9(1), 65-73 (2009). https://doi.org/10.1109/TDMR.2008.2010250

34. Wang, K., Liu, S., Chen, F., Qing, Z., Liu, Z.Y., Luo, X.B.: Freeform LED lens for rectangularly prescribed illumination. J. Opt. A Pure Appl. Opt. 11(10), 105501 (2009). https://doi.org/10.1088/1464-4258/11/10/105501

35. Yan, J.R.: Design and analysis of reflectors for non-Lambertian light-emitting diodes. Opt. Quant. Electron. 48(12), 527 (2016). https://doi.org/10.1007/s11 082-016-0804-7

36. Chaves, J.: Introduction to nonimaging optics, 2nd edn, pp. 463-464. CRC Press, New York (2015)

37. Pan, H.X., Su, Z.P., Zhang, Y.L., Wu, T.: Construct freeform surface directly in an initial layout of an off-axis reflective image system by seed curve extension and simulated annealing algorithm. J. Eur. Opt. Soc. Rapid Publ. 16(9), 1-9 (2020)

38. Shuai, Y., He, Y.Z., Tran, N.T., Shi, F.G.: Angular CCT uniformity of phosphor converted white LEDs: effects of phosphor materials and packaging structures. IEEE Photonic. Tech. L. 23(3), 137-139 (2011)

\section{Publisher's Note}

Springer Nature remains neutral with regard to jurisdictional claims in published maps and institutional affiliations. 